\title{
Integration of information in a visual discrimination task
}

\section{Z. J. ULEHLA, JOSEPH HALPERN AND ANN CERF UNIVERSITY OF DENVER ${ }^{2}$}

The integration of information model of signal detection theory (SDT) was tested in a multiple observation tilt-discrimination task. Improvement in discriminability with additional observations was consonant with theory over two observations, but observations beyond the second yielded less improvement than predicted by the integration model. Analyses of operating characteristics lent some support to the applicability of SDT to the tilt-discrimination task.

This paper describes two experiments designed to test the integration model of signal detection theory (SDT) in a multiple observation task (cf. Green \& Swets, 1966). In opposition to traditional threshold theory which posits an all-or-nothing informing effect of stimuli, SDT states that the informing effects range along a continuum from determinate information favoring one stimulus alternative through no information to determinate information favoring the other alternative. A stimulus is evaluated by comparing its informing effect with an internal standard or criterion. The probability density function of the informing effects yielded by a given stimulus source is assumed to be normal. In a two-alternative discrimination task, the informing effects density functions associated with the alternative stimuli would differ in location, but, for the sake of simplicity, can be assumed to be equal in variance. The difference between the means of the probability density functions, in units of the density function standard deviation, is labeled the index of signal detectability (d') and constitutes the SDT measure of discriminability-sensitivity.

In order to derive predictions of discriminability when multiple observations of the stimulus are furnished on each trial, it is assumed that the informing effect associated with a set of independent observations is the mean of the informing effects of the component observations. Thus, the probability density function for a set of $\mathbf{n}$ observations should possess the same location on the informing effects continuum as the density function for single observations. However, the standard deviation should be only $1 / \sqrt{n}$ ( $n$ being the number of observations) of the standard deviation of the single observation density function. Because $\mathrm{d}^{\prime}$ is the ratio of the difference in location to the standard deviation of the density functions, reduction in the standard deviation yields a proportional increase in $d^{\prime}$. Thus, the $d^{\prime}$ based on $n$ observations per trial is predicted by the SDT integration model to be $\sqrt{n}$ times the $d^{\prime}$ based on one observation per trial. The $\sqrt{n}$ prediction requires the assumption that the multiple stimulus observations are mutually independent. If dependence should exist, the predicted improvement would be less than $\sqrt{n}$. It may be noted that the same predictions can be derived if one assumes that $S$ bases his judgment on the likelihood ratio of the informing effects, and that the likelihood ratio of a set of stimulus observations is the product of the likelihood ratios of the individual observations (Green \& Swets, 1966).

In an auditory detection task, Swets and Birdsall (1967) found that the $\sqrt{n}$ prediction of the integration model was compatible with the improvement in $d^{\prime}$ with multiple stimulus observations under some response conditions but not under others. The crucial aspect of the response appeared to be whether or not $S$ was trained to evaluate the probability that his judgment of the signal was correct. In one group, $\mathbf{S}$ was trained to rate each stimulus on a four-point scale, in effect expressing a degree of confidence in his impression as to whether or not the signal was presented. A second group furnished only a yes-no binary response. The improvement in $\mathrm{d}^{\prime}$ with additional stimulus observations approximated theory in the confidence rating group, but $\mathrm{d}^{\prime}$ improvement fell short of theory in the yes-no group.

Somewhat similar results have been obtained in a conceptual discrimination experiment (Ulehla, Canges, \& Wackwitz, 1967a).
The conceptual task involved judgment of which of two literature sources furnished each of a large number of brief samples of English text. In the first of two experiments testing the $\sqrt{\mathbf{n}}$ prediction, three stimulus conditions were employed: (a) a single stimulus condition, (b) a two stimulus or pairs condition, and (c) a three stimulus or triples condition. In each condition, $S$ observed an entire stimulus set (containing one, two, or three independently sampled stimuli for the singles, pairs, and triples conditions, respectively) and judged which source magazine had furnished the stimulus set. In this experiment, improvement in $d^{\prime}$ with additional stimulus observations fell well below what SDT prescribed. In the second experiment $S$ provided a discrimination judgment and a finely grained certainty estimate (a statement of confidence in his discrimination) after observing each member of each set of three stimuli. Averaging over three kinds of conceptual stimuli, the mean $d^{t}$ computed from discrimination judgments made after two stimuli were observed was 1.38 times the mean d' for judgments made after only one stimulus was observed; the mean three stimulus $\mathrm{d}^{\prime}$ was 1.71 times the mean one stimulus $\mathrm{d}^{\prime}$. Although the extreme closeness of these means to the theoretical values of $\sqrt{2}=$ 1.41 and $\sqrt{3}=1.73$ may have been fortuitous because of substantial inter-S variability, these results suggested that the re-evaluation of judgment with certainty estimation after each stimulus may result in efficient integration of information.

The Swets and Birdsall (1967) and Ulehla et al (1967) findings suggest that the integration model may describe the improvement in discriminability yielded by multiple stimulus observations when the response demanded of $S$ is sufficiently complex to induce him to make full use of the information contained in the additional observations. The research described here included two tests of the integration model in a type of visual discrimination task previously used to investigate other aspects of SDT (Ulehla, 1966). This task involved the judgment of the direction of tilt (right vs left) of lines rotated $1 / 2 \mathrm{deg}$ from the vertical as defined by an enclosing square. To enhance Ss use of stimulus information, the response conditions which were found by Ulehla et al to yield good integration of information in conceptual discrimination were adapted to the tilt-discrimination task. The first experiment evaluated integration of information over three observations per trial, the second over five observations per trial.

The rationale for the integration model derives from SDT, and thus is applicable only to discrimination tasks adequately modeled by SDT. Use of confidence ratings enabled one test of the fit of SDT to the tilted-line task to be performed. This test involved implications of SDT for the operating characteristics (OCs) yielded by the tilt-discrimination task. Analogous to the $O C$ of a statistical test, this $O C$ describes how the hit rate to one stimulus (e.g., the proportion of left-tilt stimuli judged to be left-tilt) covaries with the error rate to the other stimulus (proportion of right-tilt stimuli judged to be left-tilt) as the criterion for decision is moved over its entire range. Examination of OCs provides one of the traditional ways of evaluating the fit of SDT to discrimination data. For this analysis, the tilt judgments were combined with the certainty judgments to yield a 12 -category ordered rating scale as follows: left-tilt $-100 \%$ certainty; left-tilt $-90 \%$ certainty; ... ; left-tilt $-50 \%$ certainty; right-tilt $50 \%$ certainty; ...; right-tilt-100\% certainty. Each boundary between adjacent rating categories corresponds to a different potential location for S's decision criterion. According to SDT, the left-tilt hit rate associated with a given decision criterion would correspond to the proportion of the left-tilt density function falling on the left-tilt side of the criterion. Similarly, the right-tilt error rate associated with the same criterion would correspond to the proportion of the right tilt density function falling on the left-tilt side of the criterion. For example, if we look at the criterion separating the left-tilt $90 \%$ 
category from the left-tilt $80 \%$ category, the hit rate would include the proportion of left-tilt stimuli called either "lefttilt-100\%" or "left-tilt-90\%." This hit rate would correspond to

the proportion of the left-tilt density function falling to the left of the criterion separating the left $90 \%$ from the left $80 \%$ category. Similarly, the right-tilt error rate associated with this criterion would be the proportion of right-tilt stimuli called "left-100\%" or "left-90\%" and would correspond to the proportion of the right-tilt density function falling to the left-tilt side of this criterion. Since the density functions are assumed to be normal, the location of the criterion can be expressed as a normal deviate ( $z$ score) on either density function. Thus, the difference between the left-tilt hit rate $\mathrm{z}$ scores associated with two criterion locations must be a linear function of the difference between the corresponding right-tilt error rate $\mathrm{z}$ scores. (The $\mathrm{z}$ score differences will be equal if the two density functions possess equal variance.) The untransformed difference in left-tilt hit rate would not be a linear function of the difference between the corresponding untransformed right-tilt error rates. In the $O C$ analyses below, two nonindependent implications of the above argument for product moment correlation coefficients ( $r$ 's) are tested: (a) the $r$ between the $z$ transforms of the cumulative response probabilities will be greater than the $r$ between the untransformed cumulative response probabilities, because curvilineants should diminish the latter; (b) the $r$ between the $z$ transforms will be essentially unity. In contrast to the SDT predictions, traditional threshold theory posits a linear $\mathrm{OC}$ without $\mathrm{z}$ transformation, and thus contradicts predictions (a) and (b) for data based on one observation per trial. Confirmation of predictions (a) and (b) would support the fit of SDT to the tilt-discrimination tasks, and thus strengthen the rationale for the tests of the integration model

\section{EXPERIMENT 1}

Method

Subjects. Eight undergraduate students served as paid Ss and were run in two groups of four Ss each.

Apparatus. A Kodak Carousel slide projector fitted with an Alphax shutter was used to project the stimuli upon a white surface. Each stimulus was a $6^{1 / 2}$ in. line tilted $1 / 2$ deg from the vertical as defined by an enclosing $19 \mathrm{in}$. square in which the line was centered. Lines termed "left-tilt" were tilted $1 / 2 \mathrm{deg}$ from the vertical in the counterclockwise direction; lines termed "right-tilt" were tilted $1 / 2$ deg in the clockwise direction.

Procedure. The S's task was to discriminate the left-tilt lines from the right-tilt lines. Each $S$ was administered 400 trials. A different slide was exposed on each trial of each block of 25 trials. The first exposure of each trial followed the last exposure of the preceding trial by $10 \mathrm{sec}$. Each trial included three 1/50 sec exposures of the stimulus presented at $5 \mathrm{sec}$ intervals. After each stimulus observation $S$ indicated his judgment of tilt by writing " $L$ " or " $R$," and indicated his certainty in his judgment by writing a figure between 50 and 100 in steps of 10 . The $S$ was instructed

Table 1

Values of d' and d' Ratios: Experiment 1

\begin{tabular}{|c|c|c|c|c|c|c|}
\hline \multirow{2}{*}{$\begin{array}{c}\text { Subject } \\
\text { Number }\end{array}$} & \multirow{2}{*}{$\frac{0_{1}}{d^{\prime}{ }_{1}}$} & \multicolumn{2}{|c|}{$\mathrm{O}_{2}$} & \multicolumn{3}{|c|}{$\mathrm{O}_{3}$} \\
\hline & & $\mathrm{d}^{\prime}{ }_{2}$ & $\mathrm{~d}_{2}{ }_{2} / \mathbf{d}^{\prime}{ }_{1}$ & $\mathbf{d}^{\prime}{ }_{3}$ & $\mathrm{~d}_{3}{ }^{\prime} / \mathrm{d}^{\prime}{ }_{1}$ & $\mathrm{~d}^{\prime}{ }_{3} / \mathrm{d}^{\prime}{ }_{2}$ \\
\hline 1 & 1.78 & 2.11 & 1.19 & 2.35 & 1.32 & 1.11 \\
\hline 2 & .15 & .28 & 1.87 & .23 & 1.53 & .82 \\
\hline 3 & 1.38 & 2.01 & 1.46 & 1.88 & 1.36 & .94 \\
\hline 4 & 1.36 & 2.05 & 1.51 & 1.90 & 1.40 & .93 \\
\hline 5 & 1.75 & 2.30 & 1.31 & 2.55 & 1.46 & 1.11 \\
\hline 6 & 2.02 & 2.76 & 1.37 & 3.04 & 1.50 & 1.10 \\
\hline 7 & 1.51 & 1.91 & 1.26 & 2.11 & 1.40 & 1.10 \\
\hline 8 & 1.62 & 2.46 & 1.52 & 3.06 & 1.89 & 1.24 \\
\hline$\overline{\mathbf{x}}$ & & & 1.44 & & 1.48 & 1.04 \\
\hline SDT Predicts & & & 1.41 & & 1.73 & 1.23 \\
\hline mean without S No. 2 & & & 1.37 & & 1.48 & 1.08 \\
\hline
\end{tabular}

Table 2.

Correlation Coefficient Between Untransformed Cumulative Response Proportions $\left(\mathbf{r}_{\mathrm{un}}\right)$ and Between Their $\mathrm{z}$ Transforms (normal deviates) $\left(r_{\mathbf{z}}\right)$ : Experiment 1

\begin{tabular}{|c|c|c|c|c|}
\hline \multirow{2}{*}{$\begin{array}{l}\text { Subject } \\
\text { Number }\end{array}$} & & \multicolumn{3}{|c|}{ Observation } \\
\hline & & 1 & 2 & 3 \\
\hline 1 & $\begin{array}{l}r_{z} \\
r_{u n}\end{array}$ & $\begin{array}{r}1.00 \\
.91\end{array}$ & $\begin{array}{l}.97 \\
.87\end{array}$ & $\begin{array}{r}1.00 \\
.95\end{array}$ \\
\hline 2 & $\begin{array}{l}r_{z} \\
r_{u n}\end{array}$ & $\begin{array}{r}.96 \\
1.00\end{array}$ & $\begin{array}{r}1.00 \\
.99\end{array}$ & $\begin{array}{r}1.00 \\
.99\end{array}$ \\
\hline 3 & $\begin{array}{l}\mathbf{r}_{\mathbf{z}} \\
\mathbf{r}_{\text {un }}\end{array}$ & $\begin{array}{l}.99 \\
.95\end{array}$ & $\begin{array}{l}.99 \\
.95\end{array}$ & $\begin{array}{l}.99 \\
.99\end{array}$ \\
\hline 4 & $\begin{array}{l}r_{\mathbf{z}} \\
r_{\text {un }}\end{array}$ & $\begin{array}{l}.99 \\
.94\end{array}$ & $\begin{array}{l}.98 \\
.86\end{array}$ & $\begin{array}{l}.98 \\
.83\end{array}$ \\
\hline 5 & $\begin{array}{l}\mathbf{r}_{\mathbf{z}} \\
\mathbf{r}_{\text {un }}\end{array}$ & $\begin{array}{r}1.00 \\
.95\end{array}$ & $\begin{array}{l}.99 \\
.86\end{array}$ & $\begin{array}{l}.97 \\
.84\end{array}$ \\
\hline 6 & $\begin{array}{l}r_{z} \\
r_{\text {un }}\end{array}$ & $\begin{array}{r}1.00 \\
.90\end{array}$ & $\begin{array}{l}.97 \\
.79\end{array}$ & $\begin{array}{l}.94 \\
.81\end{array}$ \\
\hline 7 & $\begin{array}{l}\mathrm{r}_{\mathbf{z}} \\
\mathrm{r}_{\text {un }}\end{array}$ & $\begin{array}{l}.99 \\
.97\end{array}$ & $\begin{array}{l}.98 \\
.87\end{array}$ & $\begin{array}{l}.97 \\
.83\end{array}$ \\
\hline 8 & $\begin{array}{l}\mathbf{r}_{\mathbf{z}} \\
\mathbf{r}_{\text {un }}\end{array}$ & $\begin{array}{l}.99 \\
.95\end{array}$ & $\begin{array}{l}.95 \\
.81\end{array}$ & $\begin{array}{l}.95 \\
.78\end{array}$ \\
\hline
\end{tabular}

that accurate use of the certainty ratings would result in $50 \%$ of the judgments rated 50 proving correct, $60 \%$ of the judgments rated 60 proving correct, etc. The $S$ was told that all three observations of each trial were of the same slide, and that the second (third) judgment and certainty ratings should be based on both (all three) observations. In addition to a base pay of $\$ 1$ per hour, $S$ received $1 \mathrm{c}$ for each correct stimulus judgment and was fined $1 \mathrm{c}$ for each incorrect stimulus judgment. The $\mathrm{S}$ was told the correct tilt at the end of each trial.

\section{Results}

For each $S$, three $d^{\prime}$ values were computed. The first $d^{\prime}\left(d^{\prime}{ }_{1}\right)$ was computed from judgments of the first stimulus observation of each trial; the second $d^{\prime}\left(d^{\prime}{ }_{2}\right)$ was obtained from judgments based upon the first two stimulus observations of each trial, and the third $d^{\prime}\left(d^{\prime}{ }_{3}\right)$ pertained to judgments based upon all three. Theoretically, the second and third $\mathrm{d}^{\prime}$ values should be in ratio of $\sqrt{2}$ and $\sqrt{3}$, respectively, to the $d^{\prime}$ based on the first observation only, i.e., $d^{\prime}{ }_{2} / d^{\prime}{ }_{1}=\sqrt{2}$ and $d^{\prime}{ }_{3} / d^{\prime}{ }_{1}=\sqrt{3}$. As shown in Table 1 , $\mathrm{d}_{2} / \mathrm{d}^{\prime}{ }_{1}$ was close to that predicted by the integration model, but little further $d^{\prime}$ increase was yielded by the third observation. Thus, the theory is compatible with integration over two observations but not over three.

For the $O C$ analysis, $r$ 's were computed between the $z$ transforms of the left-tilt hit rates and the $z$ transforms of the right-tilt error rates. In addition, r's were computed between the untransformed rates. Only rating categories for which both cumulative proportions fell between .01 and .99 were included. With one exception, the $r$ 's based on the $\mathrm{z}$ transforms were higher than the corresponding r's based on the untransformed proportions (see Table 2), thus supporting one SDT prediction. However, several $z$ transform r's departed from unity and thus the second SDT prediction was only partially supported.

\section{EXPERIMENT 2}

Experiment 2 was similar to Experiment 1, but with three major changes. The first was made because of the possibility that the failure of Ss in Experiment 1 to achieve the theoretical degree of the integration of information over three observations may relate to a dependence of observations within trials caused by the use of the same slide for all observations within trials (although such dependence theoretically should have diminished integration 
Table 3

Values of $\mathrm{d}^{\prime *}$ and Appropriate Ratios: Experiment 2

\begin{tabular}{|c|c|c|c|c|c|c|c|c|c|c|c|c|}
\hline \multirow{2}{*}{$\begin{array}{l}\text { Subject } \\
\text { Number }\end{array}$} & \multirow{2}{*}{$\frac{0_{1}}{d_{1}^{\prime}}$} & \multicolumn{2}{|c|}{$\mathrm{O}_{2}$} & \multicolumn{3}{|c|}{$0_{3}$} & \multicolumn{3}{|c|}{$0_{4}$} & \multicolumn{3}{|c|}{$0_{5}$} \\
\hline & & $d_{2}^{\prime}$ & $\mathrm{d}_{2}^{\prime} / \mathrm{d}^{\prime}{ }_{1}$ & $d^{\prime}{ }_{3}$ & $\mathrm{~d}_{3}^{\prime} / \mathrm{d}^{\prime}$ & $d_{3}^{\prime} / d^{\prime}{ }_{2}$ & $\mathrm{~d}_{4}^{\prime}$ & $\mathrm{d}_{4}^{\prime} / \mathrm{d}_{1}^{\prime}$ & $\mathrm{d}^{\prime}{ }_{4} / \mathrm{d}^{\prime}{ }_{3}$ & $\overline{d^{\prime}}$ & $\mathrm{d}_{5}^{\prime} / \mathrm{d}_{1}^{\prime}$ & $\mathrm{d}_{5}^{\prime} / \mathrm{d}_{4}^{\prime}$ \\
\hline 1 & 1.54 & 2.00 & 1.30 & 2.08 & 1.35 & 1.04 & 2.23 & 1.45 & 1.07 & 2.23 & 1.45 & 1.00 \\
\hline 2 & .72 & .93 & 1.29 & .97 & 1.35 & 1.04 & 1.22 & 1.69 & 1.26 & 1.48 & 2.06 & 1.21 \\
\hline 3 & .74 & .81 & 1.09 & 1.10 & 1.49 & 1.36 & 1.24 & 1.68 & 1.13 & 1.24 & 1.68 & 1.00 \\
\hline 4 & 1.25 & 1.80 & 1.44 & 2.05 & 1.64 & 1.14 & 2.11 & 1.69 & 1.03 & 1.95 & 1.56 & .92 \\
\hline 5 & 1.26 & 1.77 & 1.40 & 1.72 & 1.37 & .97 & 1.94 & 1.54 & 1.13 & 1.97 & 1.56 & 1.02 \\
\hline 6 & .81 & 1.14 & 1.41 & 1.20 & 1.48 & 1.05 & 1.11 & 1.37 & .93 & 1.41 & 1.74 & 1.27 \\
\hline 7 & 1.00 & 1.24 & 1.24 & 1.69 & 1.69 & 1.36 & 1.96 & 1.96 & 1.16 & 1.98 & 1.98 & 1.01 \\
\hline 8 & .88 & 1.34 & 1.52 & 1.58 & 1.80 & 1.18 & 1.61 & 1.83 & 1.02 & 1.80 & 2.05 & 1.12 \\
\hline 9 & .96 & 1.59 & 1.66 & 1.78 & 1.85 & 1.12 & 1.99 & 2.07 & 1.12 & 2.11 & 2.20 & 1.06 \\
\hline 10 & .94 & 1.30 & 1.38 & 1.32 & 1.40 & 1.02 & 1.40 & 1.49 & 1.06 & 1.63 & 1.73 & 1.16 \\
\hline 11 & .51 & 1.00 & 1.96 & 1.32 & 2.59 & 1.32 & 1.22 & 2.39 & .92 & 1.46 & 2.86 & 1.20 \\
\hline (mean) & .96 & 1.36 & 1.43 & 1.53 & 1.64 & 1.15 & 1.64 & 1.74 & 1.08 & 1.75 & 1.90 & 1.09 \\
\hline SDT Predicts & & & 1.41 & & 1.73 & 1.23 & & 2.00 & 1.16 & & 2.24 & 1.12 \\
\hline
\end{tabular}

over two observations as well as over three). In Experiment 2, each observation utilized a different slide. The second major change was to increase the number of observations per trial to five in order to better assess any decline in integration after the second observation. The third major change was the offer of a bonus for accurate estimation of certainty in order to further induce $S$ to evaluate the stimulus information carefully.

\section{Method}

Subjects. Eleven undergraduate students served as paid Ss.

Apparatus and Procedure. The apparatus and procedure were the same as in Experiment 1 except for the following: the number of trials was reduced to 390 . Each trial included five stimulus observations. A different slide was projected for each stimulus observation; all five slides were of the same tilt and this was explained to $S$. The exposure time was reduced to $1 / 100 \mathrm{sec}$ to avoid a ceiling effect on $d^{\prime}$ increase. The payoff was reduced to \pm $1 / 2 \mathrm{c}$. The instructions indicated to $S$ that he would receive a bonus of $\$ 1$ if "your certainty judgments prove reasonably correct." If he asked what "reasonably correct" meant, S was told that the discrepancy between nominal certainty estimates and actual probabilities of being correct should average less than $10 \%$. The actual probability of being correct associated with each certainty estimation category (e.g., $50 \%$ category) was explained to be the proportion of the judgments assigned that certainty category which proved to be correct. In actuality, all Ss received the bonus.

\section{Results}

For each $S$, five $d^{\prime}$ values were computed, ranging from $d^{\prime}{ }_{1}$ based on only one stimulus observation per trial to $d^{\prime}{ }_{s}$ based on all five observations per trial (see Table 3). The mean value of $d^{\prime}$ increased monotonically with additional observations. As in Experiment 1, the mean improvement in $d^{\prime}$ over the first two observations was consistent with theory, but improvement over subsequent observations was less than predicted. Each successive observation after the second yielded less $d^{\prime}$ increase than the integration model specifies.

The $O C$ analysis was performed as in Experiment 1. Most r's between $\mathrm{z}$ transformed proportions were higher than the corresponding r's between untransformed proportions (see Table 4) and most $r$ 's between $\mathrm{z}$ transformed proportions were close to unity. Thus, both implications of SDT for r's received partial support.

\section{DISCUSSION}

Both experiments showed the same pattern of results for the estimates of $d^{\prime}$; mean improvement in the $d^{\prime}$ values based on two observations per trial over the $d^{\prime}$ values based on only one observation per trial matched-or very slightly exceeded-the $\sqrt{n}$ prediction of the integration model of SDT. However, $d^{\prime}$ improvement based on additional stimulus observations after the second consistently fell below the predictions. Thus, the integration model of SDT was not supported for this type of visual discrimination.

Possible post hoc explanations for the obtained results involve dependence of observations and/or perceptual or attentional decrement over observations. Due to possible organismic states relevant to attention or perceptual acuity persisting over time spans of several seconds, the effects of stimulus observations adjacent in time could be intercorrelated, leading to less than $\sqrt{\mathbf{n}}$ improvement in $d^{\prime}$. Such an explanation is complicated by the fact

Table 4

Correlation Coefficients Between Untransformed Cumulative Response Proportions ( $\left.\mathbf{r}_{u n}\right)$ and Between Their $\mathbf{z}$ Transforms (normal deviates) $\left(\mathbf{r}_{\mathbf{z}}\right)$ : Experiment 2

\begin{tabular}{|c|c|c|c|c|c|c|}
\hline \multirow{2}{*}{$\begin{array}{l}\text { Subject } \\
\text { Number } \\
\end{array}$} & & \multicolumn{4}{|c|}{ Observation } & \multirow[b]{2}{*}{5} \\
\hline & & 1 & 2 & 3 & 4 & \\
\hline \multirow[t]{2}{*}{1} & $\mathbf{r}_{\mathbf{z}}$ & 1.00 & 1.00 & 1.00 & .98 & 1.00 \\
\hline & $r_{u n}$ & .97 & .92 & .92 & .97 & .90 \\
\hline \multirow[t]{2}{*}{2} & $\mathbf{r}_{\mathbf{z}}$ & .94 & .95 & .94 & .76 & .53 \\
\hline & $\mathbf{r}_{\mathrm{un}}$ & .91 & .91 & .90 & .74 & .51 \\
\hline \multirow[t]{2}{*}{3} & $\mathbf{r}_{\mathbf{z}}$ & .99 & .99 & .99 & .99 & 1.00 \\
\hline & $\mathrm{I}_{\mathrm{un}}$ & .96 & .96 & .97 & .94 & .96 \\
\hline \multirow[t]{2}{*}{4} & $\mathbf{I}_{\mathbf{z}}$ & 1.00 & .99 & .98 & .97 & .99 \\
\hline & $r_{\text {un }}$ & .96 & .91 & .85 & .85 & .91 \\
\hline \multirow[t]{2}{*}{5} & $r_{z}$ & .98 & .97 & .97 & .98 & .97 \\
\hline & $r_{u n}$ & .91 & .86 & .85 & .88 & .82 \\
\hline \multirow[t]{2}{*}{6} & $\mathbf{r}_{\mathbf{z}}$ & 1.00 & 1.00 & 1.00 & 1.00 & 1.00 \\
\hline & $r_{\text {un }}$ & .98 & .93 & .96 & .98 & .96 \\
\hline \multirow[t]{2}{*}{7} & $\mathbf{r}_{\mathbf{z}}$ & .92 & 1.00 & .99 & .98 & .98 \\
\hline & $r_{u n}$ & .96 & .98 & .87 & .84 & .85 \\
\hline \multirow[t]{2}{*}{8} & $\mathbf{r}_{\mathbf{z}}$ & 1.00 & 1.00 & 1.00 & 1.00 & .99 \\
\hline & $\mathrm{I}_{\mathrm{un}}$ & .96 & .94 & .95 & .92 & .97 \\
\hline \multirow[t]{2}{*}{9} & $r_{z}$ & .99 & 1.00 & .98 & .99 & .98 \\
\hline & $\mathbf{r}_{\text {un }}$ & .93 & .90 & .84 & .89 & .89 \\
\hline \multirow[t]{2}{*}{10} & $\mathbf{r}_{\mathbf{z}}$ & .99 & .99 & .98 & .95 & .98 \\
\hline & ${ }^{r}$ un & .96 & .90 & .88 & .89 & .92 \\
\hline \multirow[t]{2}{*}{11} & $\mathbf{r}_{\mathbf{z}}$ & .99 & 1.00 & .99 & 1.00 & .92 \\
\hline & $\mathbf{r}_{\text {un }}$ & 1.00 & .96 & .89 & .94 & .88 \\
\hline
\end{tabular}


that integration did not fall below theory over two observations, but only over three or more. A conceivable explanation for this complication would assign to the first stimulus presentation properties of an orienting signal. During the $10 \mathrm{sec}$ time period between trials, S's orientation to the stimulus may have lapsed; Ss often did fidget or show other signs of distraction between trials. The first stimulus, possessing the same location as the subsequent stimuli, may have served to reorient $S$ to the relevant stimulus properties, especially the exact location of the critical feature of the display. With the benefit of better orientation, $S$ may have received a disproportionate amount of information during the second observation of each trial, which would lead to the suggestion of good integration over two trials in spite of dependence among the observations. The orienting effect hypothesis can similarly be combined with notions of progressive inattention, perceptual decrement, or fatigue to account for the obtained pattern of results.

It may be noted that the integration model is a derivative of SDT and is therefore unlikely that the integration model would be applicable when the more general theory is not. Consequently, the $O C$ analyses were designed to yield information concerning the fit of SDT to a tilt-discrimination task. In the areas noted above where the integration model has received support, SDT has been shown to be applicable, cf. Green and Swets (1966) in an auditory detection task and Ulehla, Canges, and Wackwitz (1967b) in a conceptual discrimination task. In the present research the $O C$ analysis provided some support for the applicability of SDT to the tilted line discrimination task. Thus, the theoretical rationale on which the integration model is based would seem applicable to tilted line discriminations even though the predictions of the integration model were not confirmed.

\section{REFERENCES}

GREEN, P. M., \& SWETS, J. A. Signal detection theory and psychophysics. New York: Wiley, 1966.

SWETS, J. A., \& BIRDSALL, T. G. Deferred decision in human signal detection: a preliminary experiment. Percept. \& Psychophys. 1967, 2, 15-24.

ULEHLA, Z. J. Optimality of perceptual decision criteria. J. exp. Psychol. 1966, 71, 564-569.

ULEHLA, Z. J., CANGES, L., \& WACKWTTZ, F. Integration of conceptual information. Psychon. Sci, 1967a, 8, 223-224.

ULEHLA, Z. J., CANGES, L., \& WACKWITZ, F. Signal detectability theory applied to conceptual discrimination. Psychon. Sci., 1967b, 8, 221-222.

\section{NOTES}

1. This research was supported by National Science Foundation Grant GB-3081.

2. Address: Department of Psychology, University of Denver, Denver, Colorado 80210 .

(Accepted for publication February 12, 1968.) 